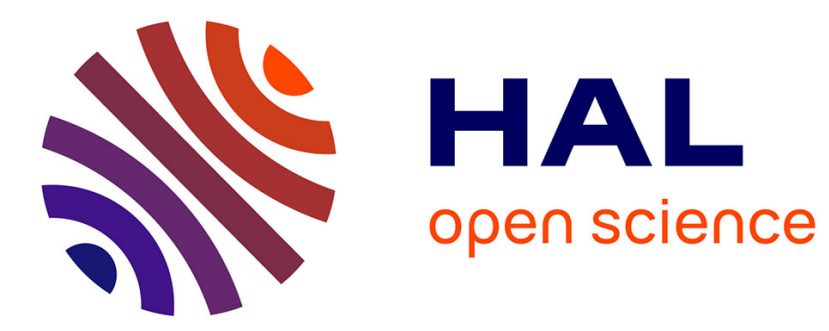

\title{
Discrete and continuous description of a three dimensional scene for quality control of radiotherapy treatment planning systems
}

Eloïse Denis, Jean-Pierre V. Guédon, Stéphane Beaumont, Nicolas Normand

\section{- To cite this version:}

Eloïse Denis, Jean-Pierre V. Guédon, Stéphane Beaumont, Nicolas Normand. Discrete and continuous description of a three dimensional scene for quality control of radiotherapy treatment planning systems. Medical Imaging, Feb 2006, San Diego, United States. pp.187. hal-00360298

\section{HAL Id: hal-00360298 \\ https://hal.science/hal-00360298}

Submitted on 10 Feb 2009

HAL is a multi-disciplinary open access archive for the deposit and dissemination of scientific research documents, whether they are published or not. The documents may come from teaching and research institutions in France or abroad, or from public or private research centers.
L'archive ouverte pluridisciplinaire HAL, est destinée au dépôt et à la diffusion de documents scientifiques de niveau recherche, publiés ou non, émanant des établissements d'enseignement et de recherche français ou étrangers, des laboratoires publics ou privés. 


\title{
Discrete and continuous description of a three dimensional scene for quality control of radiotherapy treatment planning systems
}

\author{
Eloise Denis ${ }^{a}$, JeanPierre Guédon ${ }^{a}$, \\ Stéphane Beaumont ${ }^{b}$ and Nicolas Normand ${ }^{a}$ \\ ${ }^{a}$ IRCCyN/IVC - UMR CNRS 6597, Image \& Video Communication, \\ École polytechnique de l'Université de Nantes, \\ La Chantrerie, BP 50609, 44306 Nantes Cedex 3 - France \\ ${ }^{b}$ QualiFormeD - Centre Hospitalier, \\ 85925 La Roche Sur Yon - France
}

\begin{abstract}
Quality Control (QC) procedures are mandatory to achieve accuracy in radiotherapy treatments. For that purpose, classical methods generally use physical phantoms that are acquired by the system in place of the patient.

In this paper, the use of digital test objects (DTO) replace the actual acquisition. ${ }^{1}$ A DTO is a 3D scene description composed of simple and complex shapes from which discrete descriptions can be obtained. For QC needs, both the DICOM format (for Treatment Planning System (TPS) inputs) as well as continuous descriptions are required.

The aim of this work is to define an equivalence model between a continuous description of the three dimensional (3D) scene used to define the DTO, and the DTO characteristics. The purpose is to have an XML- DTO description in order to compute discrete calculations from a continuous description.

The defined structure allows also to obtain the three dimensional matrix of the DTO and then the series of slices stored in the DICOM format. Thus, it is shown how possibly design DTO for quality control in CT simulation and dosimetry.
\end{abstract}

Keywords: CT simulation, quality control, discrete and continuous description, DTO.

\section{INTRODUCTION}

Nowadays, radiotherapy and Therapy Planning Treatment have a important role for patient treatment. Thus, it is mandatory to control the quality of softwares used in this operations. Classical methods to assess the quality of Treatment Planning System (TPS), are to use physical test objects that are acquired with a CT installation by the system in place of the patient. However the quality assessment can be more accurate using digital test objects that can be analyze directly by the TPS. These DTO are transfered to the TPS in a DICOM (Digital Imaging and Communications in Medicine) format which is a discrete image format. However, it can be interesting to have a continuous description of the shapes composing the test object, in order to control dosimetric calculation for example. Thus, a structure is defined allowing an equivalence between a continuous description of the object and a discrete version of it.

Further author information: (Send correspondence to Eloise Denis)

$a$ Email: \{eloise.denis, nicolas.normand, jean-pierre.guedon\}@univ-nantes.fr

Phone: + 332406832 47, Fax: + 33240683232

$b$ Email: stephane.beaumont@qualiformed.com

Medical Imaging 2006: Physics of Medical Imaging, edited by Michael J. Flynn, Jiang Hsieh, Proceedings of SPIE Vol. 6142, 614255, (2006) $\cdot 1605-7422 / 06 / \$ 15 \cdot$ doi: 10.1117/12.650571 


\subsection{Radiotherapy and quality control}

Radiotherapy consists in a great ionization of cancerous cells caused by radiations. This will deteriorate the genetic information composition of the cancerous cells in order to make them unable to reproduce. It is thus necessary to deliver a maximum of proportion of ionizing x-rays to the tumor while saving the maximum of neighboring healthy tissues. In order to target the tumor and to have an accurate treatment, therapy planning treatment may be used to simulate the treatment before to treat. Is it thus conformational radiotherapy. A Treatment Planning System (TPS) computes every parameter of the treatment using a virtual patient generated by a set of transverse slices acquired with a CT scanner of the patient in treatment position. ${ }^{2-4}$ This analyze of the virtual patient is called CT simulation.

CT simulation is divided in two parts. First, there is the definition of a ballistic allowing a good target covering and the lowest irradiation for normal tissues. The second part consists of the dose distribution calculation. The first step begins with the CT scan acquisition of the patient in treatment position in order to create the virtual patient. From these dataes, various calculations are computed with or without the intervention of radiotherapist :

- The doctor contours the tumor on several slices of the CT data with manual and automatic graphic tools on the TPS graphic interface. The TPS computes the volume, the contouring on each slice from this information.

- Then, there is an automatic computation of the target volume expansion. This is done in order to consider contouring uncertainties of the radiotherapist, set-up errors that can occur during the treatment, the movements of the target (internal movements and external patient movements) and beam's penumbra,

- The TPS computes the position of the isocenter, i.e. the convergence point of all beam axis.

- The next step is the computation of each beam form and orientation for the radiation. The intersection of the beams must define the target.

- Then the TPS computes some reference points to tattoo on the patient's skin to allow a pre-positioning during the treatment, and a DRR for each beam. A Digitally Reconstructed Radiograph (DRR) is a computed-generated radiograph of the virtual patient from CT data and they are used to get the patient actual positioning for treatment. ${ }^{2,5}$

All this procedure gives the treatment parameters. Thus, the accuracy of the treatment directly depend on the reliability of the TPS softwares. If the targeting does not fit well the tumor, some healthy tissues can be irradiated or some part of the tumor can not be removed. However, it is mandatory to control the quality of each algorithm of the TPS.

\subsection{Digital Test Object}

Quality assessment of TPS is classically done using phantoms i.e. Physical Test Objects (PTO) ${ }^{6-8}$ There exists a PTO for each quality criterion. ${ }^{9}$ For the quality assessment procedure, the PTO is put down on the scanner table and an acquisition of it is made. Like for a real patient, a 3D model is computed by the scanner and used by the TPS. The results of the various calculations and image computations from this data are analyzed and the quality of the TPS can be assess.

This way to control the TPS softwares quality is skewed because the controlled system is not only the TPS but the scanner and the TPS. The phantom in input of the TPS is deteriorated by the scanner acquisition and reconstruction. Thus it is difficult to know if the default on the results are due to the TPS or to the scanner. A solution to avoid this disadvantage is to use Digital Test Objects (DTO) instead of physical phantoms. ${ }^{1}$

A phantom, or test object, is an object whose features are precisely known. It is used for input of imagery systems in order to determine the characteristics of these systems. A DTO is a 3D matrix that describes the virtual object in input of the TPS. The value of each voxel of this $3 \mathrm{D}$ matrix represents the Hounsfield number of the corresponding material. A DTO is composed of 3D geometrical shapes with particular density, and behaves like the CT data of a physical phantom for the TPS. The advantage is that the geometrical properties of the phantom structure are not deteriorated at the entrance of the TPS. As the objects are not physical but virtual, 
it is easy to create as many test objects as necessary to refine the assessment of the quality if the system. For example, a quality criterion for the DRR is the divergence of each ray. A physical phantom can present only a few divergence angles to control. ${ }^{10}$ A DTO can adapt to the system it controls. In the example, every needed divergence angle can be tested with a DTO. It is easy and fast to compute a new DTO with new characteristics. Thus, the quality assessment of a system can be more precise if it is done with DTOs than with PTOs because DTO can be adapted to each system.

Another advantage of using DTOs for TPS quality control is that it is much faster. Indeed, using PTOs requires many CT acquisitions for each criteria (equivalent to 200 to 500 patients for a CT simulation software control) that takes much time and heats the scanner. Moreover, PTO are not easy to handle and to align with the CT and these problems do not exist with DTOs.

Thus, this method makes it possible to have a precise and fast quality control of TPS, avoiding the phantom damaging by the CT scanner. This method have moreover the capacity to evolve to follow the technique and the regulations. DTO are created with a particular software and the quality assessment is managed by another specific software. DTO is transfered between these two software in a DICOM format, like a regular virtual patient, but it is important to preserve the knowledge of the geometrical structure of the object, which is lost with the DICOM format. Thus, a parallel description of the DTO geometry is mandatory.

\subsection{Solid modeling}

3D solid modeling became an important problem with the development of computer graphics. The aim is to obtain an accurate representation of the shape of each object in a three dimensional scene. There exists three main representations used to model solids that are used in CAD systems mostly ${ }^{11}$ : Constructive Solid Geometry (CSG), Boundary representation (Brep) and spatial subdivision. CSG is a representation based on boolean operations of primitive shapes as parallelepiped, sphere, cylinder, cone and torus. Usual allowed boolean operations are union, intersection and subtraction. Rigid transformations as translation, scaling and rotation can be applied on each shape. The shapes are located on a global coordinate system but each one has its own coordinate system. Boundary representation represents the solids by their surfaces, edges and vertices. Spatial subdivision consists in decomposing the space into cells. In this representation, the space is divided according to a binary tree which defines a recursive subdivision of the 3D space. If a subdivision contains a shape, it is subdivided again until the unit cell. This way to represent the space is close to the voxel representation. CSG and Brep allow to manipulate features, contrary to spatial subdivision which does not contain any concept of feature. Hybrid representations can be defined as CSG-Boundary representation. For example, CSG can be improved with boundary representation for volume modeling in a discrete space. ${ }^{12}$ In this work by Emmerling et al., every operation and transformation are done in a discrete space representation. An extension of CSG is used : Constructive Volume Geometry (CVG) in which objects are represented by their scalar fields. ${ }^{13}$ In CVG, shapes can be defined by a mathematical scalar field as well as a discrete volume dataeset. Discrete and continuous shape representations are mixed into a hierarchical structure. CSG can moreover be improved with free-form modeling tools to sculpt virtual objects. ${ }^{14}$

Boundary representation can be used for solid modeling. The most usual way to represent object boundaries is the use of polygon meshes. The shapes surfaces are composed of polygons (that are frequently triangles). These polygons are formed by connecting vertices. An other way to represent object boundaries is implicit surfaces where surfaces are defined by functions. Nurbs and Point-based modeling ${ }^{15}$ are continuous examples of

this representation. A discrete representation of surfaces is defined by Bertrand ${ }^{16}$ by inter-pixel surfels, lignels and pointels. Discrete surfaces can be obtained like continuous ones with a marching-cubes like algorithm. ${ }^{17} \mathrm{~A}$ reversible polygonalization of these discrete surfaces is moreover defined by Sivignon. ${ }^{18}$ It is moreover possible to compute boolean operations on solids represented by their boundaries as Adams and Dutré show in their work. ${ }^{19}$

\section{METHODS}

The aim of this work is to obtain a data structure framework to store the digital test objects in a way that there is an equivalence between a discrete and a continuous version of it. The DTO can be translated into a series of 
DICOM files like a series of CT slices, but the same description leads to a unique continuous description of the three dimensional scene that will be exploited with assessment purposes.

The XML format used to store the DTO with its structure characteristics is now described. Then, the file structure describing a DTO is explained. Finally the method to obtain a discrete version of a DTO from its continuous description is shown in the last part of this section.

\subsection{XML format}

Extensible Markup Language (XML) is a text format derived from SGML (Standard Generalized Markup Language). It is a World Wide Web Consortium standard. ${ }^{20}$ XML is not a language but a meta-language which is used to create flag languages that can be used to describe all kinds of structured data and texts. Every XML user can define his own flags and create his own structure language. That represents an obvious interest to use this format to describe DTOs since a rigid structure can be created specially for our needs in terms of medical imaging quality control.

An XML file is structured in "elements" using "tags" which mark the beginning and the end of each element. An element can contain text or other elements. The whole XML document data is contained in a single element called "root". Each element can have attributes in order to precise its contents.

The structure of an XML file can be formally described. A simple text data structure is defined into a DTD (Document Type Definition) which is associated with the type of file. A more complex structure of data of multiple nature is defined in a XML Schema Description (XSD). A XSD file is a XML file and can be manipulated like any other XML file. This structure definition file allows for specifying each data type contained in the XML file. This point is important for a geometry definition because it is mandatory to check if each parameter is really an integer or a float, or any other type of data.

Another advantage of using a XML format to describe DTO lies in the fact that its extensive uses have changed the databases format and access.

\subsection{Defining a DTO with the specific XML format}

A specific XML format was designed to describe DTOs. This format is divided into two parts. The first one contains general data on the three dimensional scene and the second one contains a description of each geometrical shape forming the DTO. The two parts are XML elements and are children of the root element "DTO".

\subsubsection{General data of the DTO}

The first part contains all data of the DTO that is not a geometrical shape. This information is mandatory to convert the DTO in DICOM RT format. ${ }^{21}$ The DICOM format not only contains an image but information about the patient and the treatment. The $3 \mathrm{D}$ volume of the virtual patient is described by a series of DICOM 3 files. In addition, the DICOM format for radiotherapy (RT) is composed by three types of files : RT-Image, RTstructure and RT-Plan. DRRs are stored in RT-image files. General and geometrical information about patient and virtual patient are to be find in this file. RT-structure files contain informations on outlined structures and skin-marking, and RT-Plan files contain all beams parameters for the treatment. All the information required for DICOM RT is stored in this XML file part, like for instance slice thickness, space between slices and image resolution.

\subsubsection{Geometrical data of the DTO}

The second part of the XML file contains the geometrical structure of the DTO. The DTO three dimensional scene is made up of 3D shapes of different densities i.e. the Hounsfield number of the material they represent. The shapes can be combined to make more complex shapes. Thus they have to be ordered in a structure. The easiest way to do it is to arrange them into a tree whose root is the DTO itself. The tree leaves are primitives forms. The nodes of the tree are combinations of shapes. The tree nodes are combinations of shapes. A node contains an operator and the operands are the children of the node. It is thus possible to generate complex shapes from primitives and from other complex shapes. Each node or leave is a shape intervening in the construction of the test object. They consequently have an associated density. This density is an attribute of the node. For a 
complex shape, the densities of operand shapes are not taken into account but only that of the node representing the combination of shapes. Each shape, complex or primitive, can undergo transformations. Transformations are operations on a shape that modify it. They are attributes of shapes nodes (leaves or not) in the tree.

Only few primitives are mandatory to design a DTO for radiotherapy TPS quality control. Each shape has a reference point that allows to locate it in the $3 \mathrm{D}$ oriented space. Before any transformation, the reference point of a primitive is centered on the origin. The shapes are referred by their name which is noted as an attribute of the node.

Parallelepiped The first primitive shape is a parallelepiped. Initially, its edges are parallel with the axis of the coordinate system. The reference point of this primitive is the corner with the smallest coordinate in every direction. Only three parameters are thus necessary to define a parallelepiped : the three dimensions on each direction. These dimensions are constrained to be integers in order to make it easier to have a discrete description of the shape.

Ellipsoid The second available primitive shape is en ellipsoid. It is defined from three radii. The radii are the lengths along each axis of the coordinate system. If they are equal, the shape is a sphere. The reference point of this primitive is its center.

Conical frustum with elliptic base The last primitive shape is a conical frustum with elliptic base. A conical frustum is a truncated cone created by slicing the top of the cone with a cut made parallel to the base. The cone is only defined by two radii. Thus, its base is not necessarily circular but can be elliptic. This primitive is defined by its height (constrained to be integer in order to make it easier to have a discrete description of the shape), the two radii of the first base and one radius for the second (the second radius can be deduced proportionally from the three others). If these four radii are equal, the shape becomes a cylinder. The reference point of this shape is the center of the first base. The first base is the one with the smallest coordinate in the coordinate system.

It is possible to combine primitive shapes in order to obtain complex shapes. Three shapes operations are available : intersection, union and subtraction. A complex shape can be a combination of primitives or of already computed complex shapes. It is possible to combine primitive shapes in order to obtain complex shapes. Three shapes operations are available : intersection, union and subtraction. A complex shape can be either a combination of primitives or of already computed complex shapes. A shape can undergo some rigid transformations. Available transformations are translation and rotation. No homothety is available because it is not useful in DTO design. Even complex shapes can undergo transformations. Translations are made along the coordinate system axis with integer values. Rotations are made around the axis of the coordinate system with the origin as an invariant point. They are written down in degrees in the XML file. Rotations and translations do not commute. A rotation depends on an invariant point. A shape can rotate around the origin or around its own point of reference. The chosen method for shape rotation is to center it on the origin of the coordinate system. If the rotation is computed before any translation, the shape rotates around its reference point. Thus a first rotation is allowed on shapes before any translation. This is not sufficient to design DTOs. It is interesting to have a rotation after a translation in order to obtain a circle of shapes (for contrast resolution of DRR computation control for example) as shown in figure 1 . This permits to have shapes at exactly the same distance of a given point (that can be the isocenter during quality assessment). Thus the shape elements of the XML file contain two series of rotation attributes : the first one, which is applied before translations, is named "rotIntern" and the second, which is applied after translations, is named "rot".

An example of DTO structure can be seen at figure 2. This DTO can be used to control the divergence of ray casting in DRR computation in the same way as Craig et al. phantom. ${ }^{10}$ 


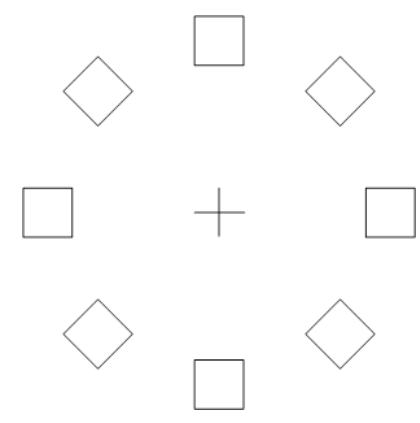

Figure 1. Example of shapes rotation. The first shape is created and duplicated seven times. Each shape is rotation with a different angle around the origin.

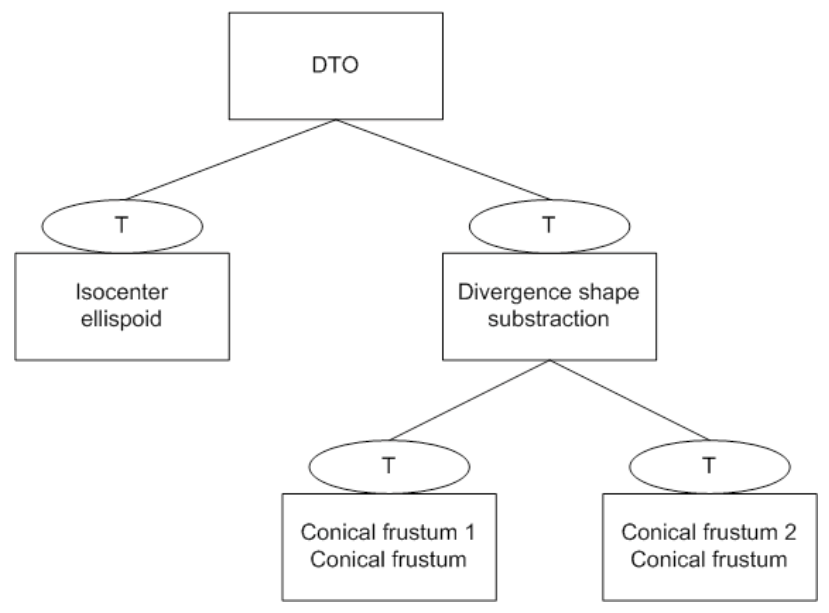

Figure 2. Shapes tree of a DTO than can be used to control ray divergence in digital reconstructed radiograph computation.

\subsection{Shapes discretization}

When the DTO is translated into a series of DICOM files, the continuous description of the 3D scene is converted into a three dimensional matrix. Thus each voxel of the 3D matrix must correspond to one and only one shape in order to attribute a value to the voxel. For sake of simplicity, voxels are consider as a uniform unit volume. Hence, they belong to a shape or not, but they do not belong partially to it. For that purpose, the voxels of the DTO are traversed and for each voxel a value, or shape membership must be determined.

The center of the voxel whose value is being determined has discrete coordinates in the 3D space. The method consists on traversing the tree that contains the shapes and looking for each node if the voxel belongs to the shape. A shape can be included into another one. Thus, the way to traverse and construct the tree is important. The route is made in the width of the tree. If a shape is included into another, it must be encountered in a second time during the route. In this way, the value of the voxel will be the one of the included shape.

Thus, it is mandatory to have some function determining if a voxel belongs to a shape or not. For a complex shape, its subtree should be studied as follows: For a complex shape, its subtree should be studied as follows:

- if the shape is a union between two shapes, the voxel belongs to it if it belongs to almost one subtree shape,

- if it is an intersection, the voxel belongs to the shape if it belongs to the two shapes of the subtree,

- if it is a subtraction, the voxel belongs to the shape if it belong to the first subtree shape and not to the second one. 


\subsubsection{Primitives discretization}

It is quite easy to determine if a voxel belongs to a parallelepiped or not. Parallelepiped were constrained to have integer dimensions and they are aligned with the discrete grid. They are forced to have an integer number of voxels in each direction for an obvious sampling (it is also easy to know if a voxel belongs to a parallelepiped if there are no rotation apply on it).

To know if a voxel belongs to an ellipsoid, it is necessary to calculate the distance between the center of the ellipsoid and the voxel. If this distance is inferior to the radius (in a direction), the voxel belongs to the shape. Two questions comes up. The first one is about the choice of the distance that must be used. A discrete distance like the Chamfrein distance is useful for distance map calculation. It is not here interesting to have such properties. The most appropriated distance is the Euclidean distance. Accordingly to the ellipsoid equations, a point belongs to an ellipsoid if:

$$
\sqrt{\frac{\left(x-x_{0}\right)^{2}}{R_{x}{ }^{2}}+\frac{\left(y-y_{0}\right)^{2}}{R_{y}{ }^{2}}+\frac{\left(z-z_{0}\right)^{2}}{R_{z}{ }^{2}}} \leq 1,
$$

where $x, y$ and $z$ are the point coordinates, $x_{0}, y_{0}$ and $z_{0}$ the ellipsoid center coordinates, $R_{x}$ is the length along the $\mathrm{X}$-axis, $R_{y}$ is the length along the $\mathrm{Y}$-axis and $R_{z}$ is the length along the $\mathrm{Z}$-axis. The second question is about the choice of the point coordinate to represent the voxel. Does a voxel must be considered in the ellipsoid if only a part of it verify the equation 1 or if the whole volume that it describes verify this equation? There are two opposite cases: the farthest voxel's point from the center of the ellipsoid is taken for the distance calculation in equation 1 or the nearest voxels point from the center is taken. In the first case, the volume of the discrete ellipsoid will be decreased compared to the volume of the continuous ellipsoid and in the second case, the discrete volume will be increased. An intermediate solution is to take the center of the voxel to refer it. Thus, $x, y$ and $z$ in equation 1 are the voxel center coordinates.

Determining if a voxel belongs to a conical frustum is done in two steps. A conical frustum primitive is constrained to have an axis parallel to the Z-axis of the coordinates system. Thus, this shape can be seen like a stack of ellipse. The height of the shape is forced to have an integer value. So, it has an exact number of voxels along the height. The discrete shape can be define like a stack of discrete ellipse. The 3D problem of discretization is thus reduced to a series of $2 \mathrm{D}$ discretizations. If the Z-coordinate of the voxel corresponds to a discrete ellipse of the conical frustum, the voxel belongs to the shape if the distance to the center of the ellipse is inferior or equal to the radius. As the the ellipsoid case, the Euclidean distance is chosen and the center of the voxel is taken as the voxel reference point. Thus, accordingly to the ellipse equations, a point belongs to an ellipse if:

$$
\sqrt{\frac{\left(x-x_{0}\right)^{2}}{R_{x}{ }^{2}}+\frac{\left(y-y_{0}\right)^{2}}{R_{y}{ }^{2}}} \leq 1,
$$

where $x$ and $y$ are the center of the voxel coordinates in the plane $(X, Y), x_{0}$ and $y_{0}$ the ellipse center coordinates in the plane $(X, Y), R_{x}$ is the length along the $\mathrm{X}$-axis, $R_{y}$ is the length along theY-axis.

\subsubsection{Transformations}

Translation are constrained to be along the axis of the coordinates system and with integer values. Thus, they do not influence the discretization problem. Rotations give shapes that are not necessary aligned with the discrete grid. . To know if a voxel of a DTO belongs to a shapes that underwent transformations, the method is to considerate the coordinate of the center of this voxel, and apply to it the inverse transformations in the opposite order. New coordinates are obtained and the voxel belongs to the shape if the new point belongs to the continuous shape (see 2.3.1). In homogeneous coordinates, geometrical transformations are computed by matrix multiplication. Let $R_{1 x}, R_{1 y}$ and $R_{1 z}$ the rotation matrices for the first rotation, $T$ the translation matrix and $R_{2 x}, R_{2 y}$ and $R_{2 z}$ the rotation matrices for the first rotation. If they are applied to the shape in this order, the new point coordinates are :

$$
\left(\begin{array}{c}
x^{\prime} \\
y^{\prime} \\
z^{\prime} \\
1
\end{array}\right)=R \cdot\left(\begin{array}{l}
x \\
y \\
z \\
1
\end{array}\right)
$$


where $x, y$ and $z$ are the center of the voxel coordinates, $x^{\prime}, y^{\prime}$ and $z^{\prime}$ are the new coordinates and $R$ is defined in equation 4 :

$$
R=R_{2 z}^{-1} R_{2 y}^{-1} R_{2 x}^{-1} T^{-1} R_{1 z}^{-1} R_{1 y}^{-1} R_{1 x}^{-1} .
$$

This method to constrain many parameters to have integer values can not permit a precise shape construction. However, the voxel size (in millimeters) is require to translate the scene in DICOM format. Thus, to have a more precise shape definition, it is just necessary to reduce the voxel size.

\section{RESULTS}

In this part, a very simple DTO is presented in order to illustrate the method of DTO description. The chosen DTO is a simple phantom that can be used to control the beam divergence in digital reconstructed radiograph computation. A DRR is a virtual radiograph of the virtual patient. For each pixel of the resulted digital image, a ray is casted from the center of the pixel to the source in the 3D matrix of the virtual patient. Along this ray, the theoretical ray attenuation by the crossed materials is calculated. The rays are casted in a cone beam geometry. Thus it is mandatory to control the divergence of this cone beam. This divergence can be controlled with a phantom that contains a shape with the same divergence as the beam one.

The DTO that illustrates the work is made accordingly. It is not a final DTO but a very simple one that show the way a DTO can be build in TPS quality control. This DTO is composed of a hollowed out conical frustum with an ellipsoid base (which is a divergent shape). This phantom can be used calculating a DRR of it positioning the beam source at the top of the cone used to build the frustum. If the the beam of the DRR computation is perfect, only a thin ellipse is seen in the resulted DRR. If not, the stroke is thicker. The conical frustum does not have a circle base but an ellipsoid one. Thus, many divergence angles can be tested.

The DTO is made of a complex shape for the divergence shape and a small sphere representing the isocenter (see 2). The complex shape is a subtraction between two conical frustums. Both have a height of 151 voxels (along Z-axis). The radii of the principal basis of the first frustum are 100 (along X-axis) and 50 (along Y-axis) (the unit is the length of a voxel edge). The radius along $\mathrm{X}$-axis for the second basis is 50 , and the radius along Y-axis that is calculated from the three other ones is 5 . The second conical frustum is a little thinner. Its radii are 98 (along $\mathrm{X}$-axis) and 49 (along Y-axis) for the principal basis, and 9 (along X-axis) and 4.5 (along Y-axis) for the second basis. By subtracting the second frustum from the first one, the complex shape for the divergence control can be obtained. The density of this shape is 1024, in Hounsfield number.

The DTO is named DivergenceDTO and its size is $256 \times 256 \times 256$ voxels. A series of parameters allows to locate the DTO in the 3D space coordinate system. Each voxel is a unit cube whose sides are one millimeter length. Each corresponding CT slice is one voxel width and zero voxel between each. Consequently, there will be 256 slices for this DTO. The background density of this DTO is -1024 , in Hounsfield number. The software to convert the DTO in DICOM files allow an image storage onto 12 or 16 bits. The XML tag "storage" specify which option to choose for the DICOM conversion. Then, a description of the DTO is stored in the XML file. This describe the procedure to control a quality criterion with this phantom and how the result should be. Follows a series of tags specific to DICOM format requirements.

The following XML file is obtained from the previous DTO specifications:

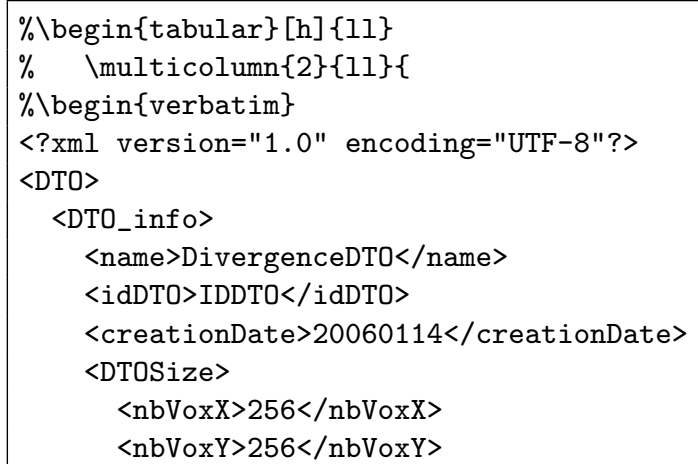




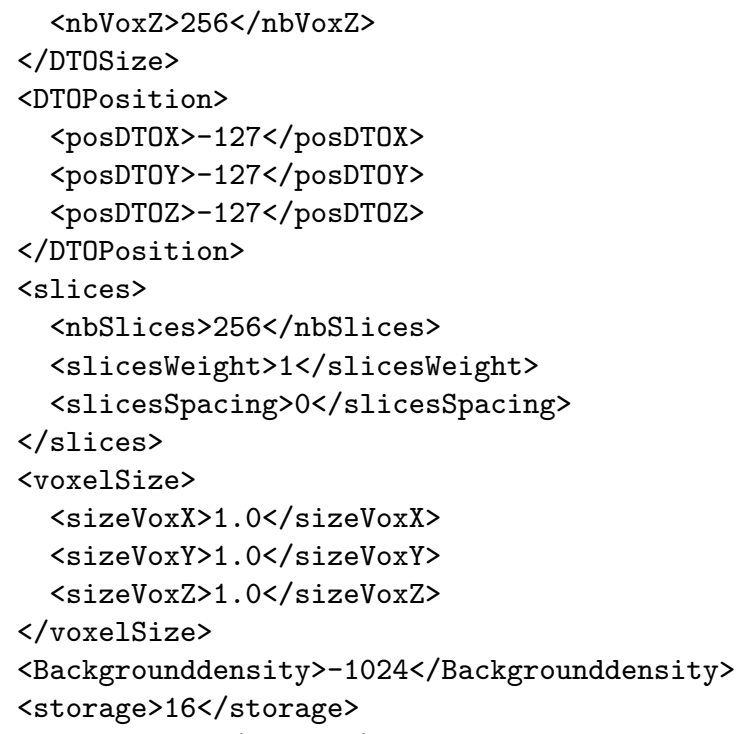




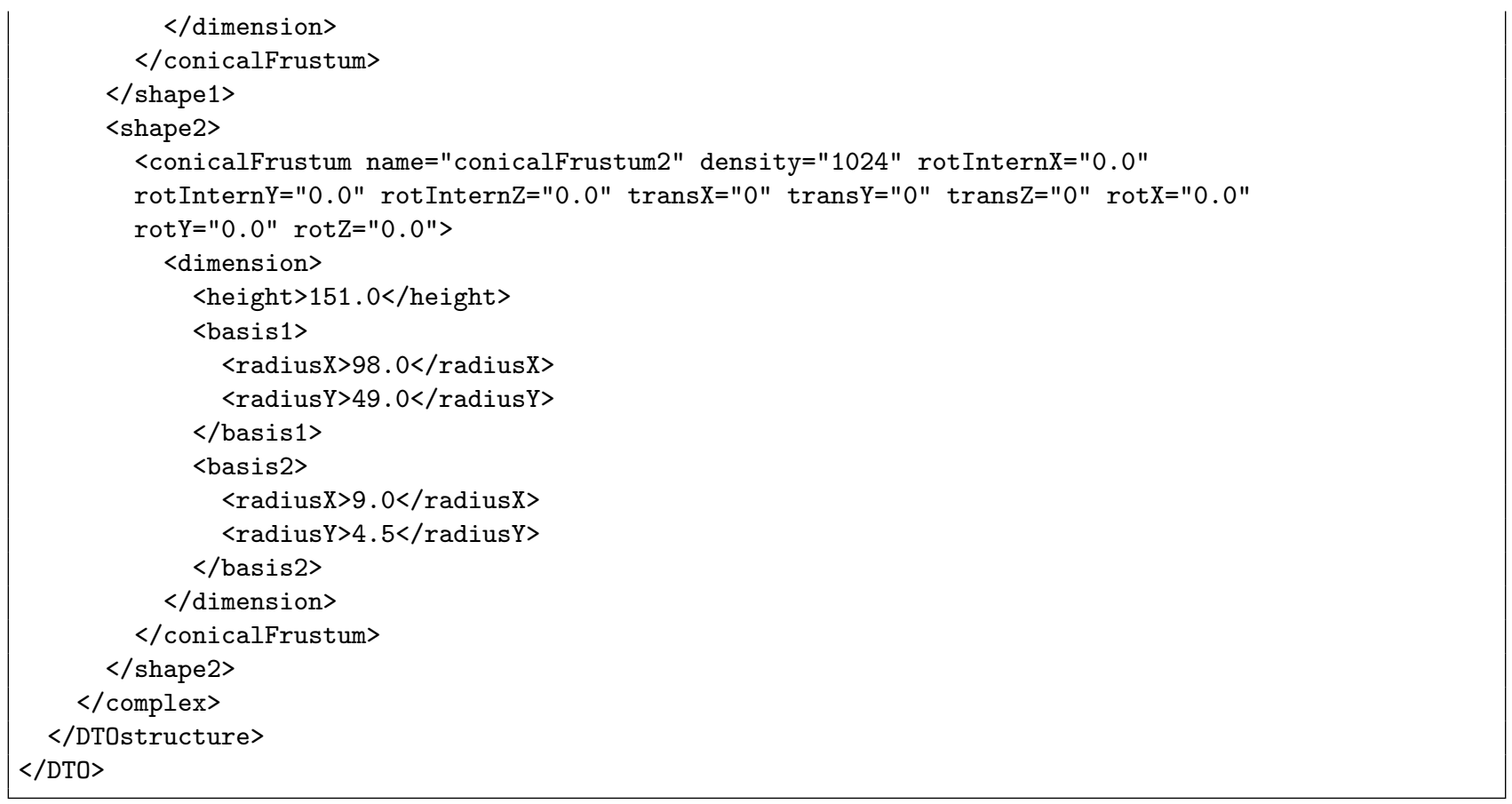

A series of DICOM CT slices are computed from this DTO XML file. Three of them are presented at figure 3. These images are in conformity with the DTO description. The divergence of the shape can be checked and slices of a hollowed out ellipsoidal conical frustum can be recognized. The series of DICOM files can be transfered to the TPS in order to control the beam divergence in DRR computation.

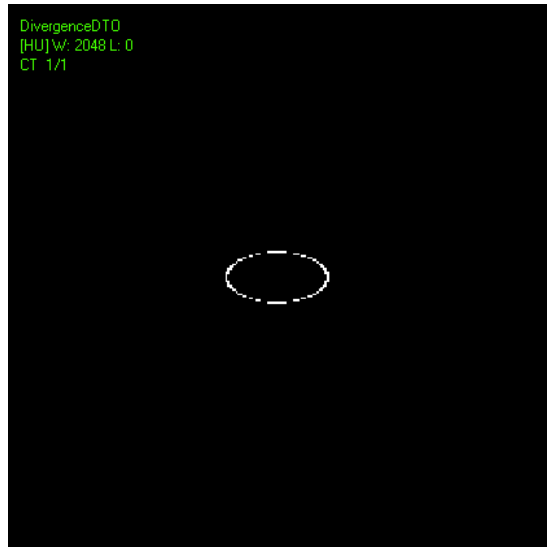

(a) This slice is near the beam source, thus the ellipsoid is small.

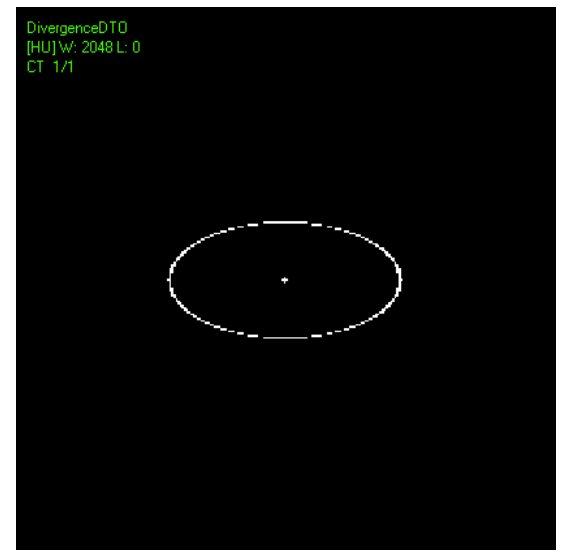

(b) This slice is in the middle of the DTO. The mark for the isocenter can be seen in the center of the image.

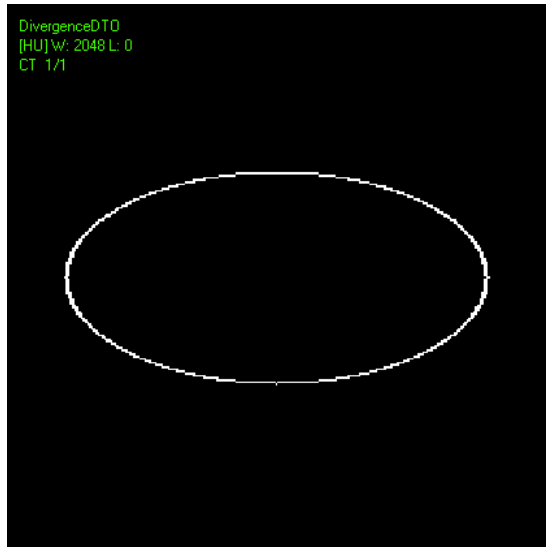

(c) This slice is far from the beam source, thus the ellipsoid is big.

Figure 3. DICOM slice image of the divergence DTO.

\section{APPLICATIONS}

The target application is the quality control of radiotherapy devices. The presented framework will allows for the DTO creation, the DTO storage, the DTO experiments and the DTO results analysis without any kind of error due to the device acquisition or to the object sampling. 


\subsection{DTO storage for DTO creation software}

The method to store 3D scene in XML format, specifically for digital test objects can be employed to store DTO during their creation in a specific software. All the information is here to translate into DICOM format to obtain all images of a virtual phantom for treatment planning systems quality assessment. A DTO can thus be modified much more easily than if it was only saved in DICOM because the geometrical structure information is recorded. For instance, if the size of a shape has to be changed in order to make the control more precise, the scene has to be created again from scratch if only the DICOM version had been stored. With the XML file, only a parameter have to be changed and the DICOM files can be easily computed.

\subsection{Information for quality control tests}

The software to manage the quality assessment with DTO is being developped and tested. The phantom used in input of the TPS corresponds to the DICOM version, as a series of CT slices. The role of this software is to manage the scene under test, the phantom characteristics and the computation for each test between the result of the operation made by the software under test operating onto the phantom on one side and the corresponding DTO on the other side. All these information are not stored in the DICOM slices but in the XML file.

\section{CONCLUSION}

In this paper, a description of digital test objects has been defined. This description allows for a continuous representation of the phantom and its equivalent discrete representation can be obtained. DTO's three dimensional scene were defined into a hierarchical tree structure. The leaves are primitives and the nodes correspond to complex shapes obtained by boolean operations. Rigid transformations are applied on each shapes. XML format was chosen to store this continuous description. The file is divided into two parts. The first one contains general DTO data, mostly for DICOM translation. The second part correspond to the shape definition tree. A method to obtain a discrete version of the DTO was exposed. The corresponding digital discrete phantoms can

be translated into DICOM files and sent to treatment planning systems for quality control.

This continuous description of digital phantoms is also adapted to translate DTOs into PENELOPE format. Indeed, a future work will be to create a platform to convert both PENELOPE continuous phantom descriptions to DICOM discrete files and, to convert discrete phantoms to PENELOPE format. The DICOM CT files do not represent a sufficient information to perform this conversion, whereas the XML DTO description is able to do it. This DTO conversion to PENELOPE format will allow to perform the dosimetric calculation quality control.

\section{REFERENCES}

1. E. Denis, S. Beaumont, and J.-P. Guédon, "Digital reconstructed radiography quality control with software methods," in Proceedings of SPIE, Medical Imaging 2005: Physics of medical imaging, M. Flynn, ed., 5745, pp. 1002-1013, SPIE, 2005.

2. L. Coia, T. Schultheiss, and G. Hanks, eds., A Practical Guide to CT Simulation, Advanced Medical Publishing, Madison, WI, 1995.

3. S. Beaumont, "New requirements and technology in virtual simulation," Physica Medica XIX(3), p. 245, Jul-Sept 2003.

4. A. Beaudre and A. Pica, "Simulation virtuelle : moyens et méthodologie," Cancer/Radiother 1, pp. 573-580, 1997.

5. M. Goitein and M. Abrams, "Multidimensional treatment planning: II. beam's eye view, back projection, and projection throughout CT sections," Int. J. Radiat. Oncol. Biol. Phys. 9, pp. 789-797, 1983.

6. S. Mutic, J. Palta, E. Butker, I. Das, M. Huq, L.-N. Dick Loo, B. Salter, C. McCollough, and J. Van Dyk, "Quality assurance for computed-tomography simulators and the computed-tomography-simulation process: Report of the AAPM Radiation Therapy Committee Task Group No. 66," Med. Phys. 30, pp. 2762-2792, October 2003. 
7. P. Andreo, J. Cramb, B. Fraass, F. Ionescu-Farca, J. Izewska, V. Levin, B. Mijnheer, J.-C. Rosenwald, P. Scalliet, K. Shortt, J. Van Dyk, and S. Vatnitsky, "Commissioning and quality assurance of computerized planning systems for radiation treatment of cancer," Tech. Rep. 430, International Atomic Energy Agency (IAEA), 2004.

8. B. Fraas, K. Doppke, M. Hunt, G. Kutcher, G. Starkschall, and J. Stern, R. Van Dyke, "American Association of Physicists in Medicine Radiation Therapy Committee Task Group 53: Quality assurance for clinical radiotherapy treatment planning," Med. Phys. 25, pp. 1773-1829, October 1998.

9. T. Craig, D. Brochu, and J. Van Dyk, "A quality assurance phantom for three-dimensional radiation treatment planning," Int. J. Radiat. Oncol. Biol. Phys. 44(4), pp. 955-966, 1999.

10. T. Craig, D. Brochu, and J. Van Dyk, "A quality assurance phantom for three-dimensional radiation treatment planning," Int. J. Radiat. Oncol. Biol. Phys. 44(4), pp. 955-966, 1999.

11. F. Jara, "About solid modeling by using feature-based models." University of Massachusetts at Lowell, Computer Science Department, 1996. Computer Graphics course.

12. A. Emmerling, K. Hidebrand, J. Hoffman, P. Musialski, and G. Thurmer, "A system for modelling in threedimensional discrete space," in DGCI, Lecture Notes in Computer Science 2886, pp. 534-543, Springer, November 2003.

13. M. Chen and J. V. Tucker, "Constructive volume geometry," Computer Graphics Forum 19(4), pp. 281-293, 2000 .

14. J. Andreas Boerentzen, "Octree-based volume sculpting." Technical University of Denamark.

15. M. Reunanen, "Point-based modeling." Helsinski University of Technology, 2004.

16. Y. Bertrand, C. Fiorio, and Y. Pennaneach, "Border map: A topological representation for nd image analysis," in DGCI, Lecture Notes in Computer Science 1568, pp. 242-257, Springer, March 1999.

17. X. Daragon, M. Croupie, and G. Bertrand, "Discrete frontiers," in DGCI, Lecture Notes in Computer Science 2886, pp. 236-245, Springer, November 2003.

18. I. Sivignon, F. Dupont, and J.-M. Chassery, "Reversible polygonalization of a 3D planar discrete curve: Application on discrete surfaces," in DGCI, Lecture Notes in Computer Science 3708, pp. 347-358, Springer, September 2005.

19. B. Adams and P. Dutré, "Interactive boolean operations on surfel-bounded solids," ACM Transactions on Graphics (TOG) 22, pp. 651-656, July 2003.

20. World Wide Web Consortium, "http://www.w3.org/xml/."

21. National Electrical Manufacturers Association, "Digital Imaging and Communications in Medicine (DICOM)," 2004. 